INTERNATIONAL DESIGN CONFERENCE - DESIGN 2018

https://doi.org/10.21278/idc.2018.0222

\title{
INTEGRATING THE KNOWLEDGE ABOUT FUNCTIONAL INTERDEPENDENCIES INTO A PARAMETER MANAGEMENT APPROACH
}

\author{
F. Toepfer, T. Naumann, J. Anderer and S. Vajna
}

\begin{abstract}
Based on an approach which uses the description of functional interdependencies to resolve multicriterial goal conflicts this paper addresses the challenge of formalizing the complex knowledge about functional interdependencies and making this knowledge broadly retrievable and applicable in engineering processes. Therefore a second approach, designed to foster transparency and consistency of engineering parameters, is used to integrate this knowledge and to make it applicable based on a concept of target value management and information flow.
\end{abstract}

Keywords: complex systems, systems engineering (SE), knowledge management, parameter management, solution spaces

\section{Introduction}

Based on the understanding of Product Development as a functionally highly differentiated and interrelated sociotechnical system (Ropohl, 2009; Naumann, 2005), managing its complexity poses a key challenge that has been subject to extensive research in the field of Systems Engineering (e.g. Haberfellner, 2012; Naumann, 2016). In a technologically advancing environment increasing requirements in products have subsequently led to more advanced and complex technical solutions. Equally the complexity within Engineering has drastically increased as a growing number of functional requirements and technologies are a cause for the differentiation and distribution of tasks at a larger scale. In order to synthesize an increasing quantity and manifoldness of highly interrelated Engineering Objects (in this paper referred to as the entity of structured information represented by heterogeneous graphical formats, in partial models of an integrated product- and process- model (Bitzer et al., 2007; Faisst and Dankwort, 2007)) to a fully integrated and feasible product engineers have to communicate and coordinate their work continuously. Increasing efforts in coordination and communication hence reflect the coherent level of social complexity in engineering. At a level where social and technical complexity surpasses a limited psychological capacity it becomes unmanageable for the individual and requires the application of appropriate tools and methods (Hacker, 2002; Dörner, 2003).

Accordingly this paper addresses the challenges of communication and coordination in a complex Engineering environment and therefore firstly focusing on two questions:

- How can information about Engineering Objects be communicated effectively within the Engineering Organization?

- How can goal conflicts resulting from contradictory requirements be coordinated and settled effectively between different responsibilities? 
Generally, tools capable of automating such tasks have to manage interdependencies between Engineering Objects to some extent. Due to the huge variety of Engineering Objects and interdependency-types the informational content which is managed in such tools depends on the method, the scope and the granularity of the underlying data model. As the acceptance of such tools largely depends on the usability, the broad applicability and the effort required to generate and maintain its informative value, the scientific discourse about suitable approaches and their scope of application has been ongoing (e.g. Browning, 2001; Estefan, 2007; Dickerson and Mavris, 2013).

For any such approach it has to be taken into consideration that it requires the explicit formalization of interdependencies in a suitable model first. For complex functional interdependencies particularly this task can only be done by few individuals as it requires expert knowledge and tools capable of properly depicting inherent relations. However, once formalized this knowledge is often not accessible for nonexperts as it requires the comprehension of the underlying model and the expert tool itself $-\mathrm{a}$ circumstance most frequently not given.

As the consideration of functional and structural interdependencies are essential for the coordinationand decision-making process in engineering (Gopsill et al., 2016) it has to be the aim to make the formalized knowledge retrievable and applicable for a broad range of engineers. This includes the knowledge about already determined feasible solutions.

The research question that is therefore addressed is: How can the knowledge about functional interdependencies and information about feasible solutions be formalized and made retrievable in order to be used in complex engineering processes?

Based on an approach which uses the description of functional interdependencies to resolve multicriterial goal conflicts (Section 2) and an approach which manages systemic interdependencies on a parameter level (Section 3) this paper describes a concept which allows the integration of formalized functional interdependencies and reconciled solutions based on engineering parameters (Section 4). The approach presented in Section 2 is currently implemented as prototype for the application in the early functional vehicle layout at Daimler AG, Germany. Based on the approach presented in Sections 3 which has been enrolled for productive use in the vehicle development processes at Daimler AG the concept described in Section 4 is currently implemented prototypically.

\section{Formalization of functional interdependencies to resolve goal conflicts}

\subsection{Challenges in the coordination of goal conflicts}

A common approach in the development of complex systems is the decomposition into smaller manageable subsystems or respectively the decomposition of system functions (Dickerson and Mavris, 2013). Although decomposition is an effective enabler for the distribution and specialization of tasks it inevitably leads to interface problems between decomposed entities. Subsystems and sub functions have to be reconciled with one another to allow for a coherent integration.

Based on the understanding of functions as relational characteristics of a system (DIN 2330, 1996; Ehrlenspiel and Meerkamm, 2013) it is possible to influence system functions through the design of qualitative characteristics of system elements hereafter referred to as design variables. Once design variables are subject to multiple functions goal conflicts may arise due to contradictory requirements of the functions. Depending on the level of interconnectivity of design variables the difficulty in the coordination between different disciplines increases. As outlined by Eichstetter et al. (2014) the layout of design variables which are subject to multiple functional requirements traditionally takes place in a sequential process (Figure 1 (a)). Design variables are adjusted iteratively from different disciplines to best fulfil discipline-specific requirements. This procedure however only considers single solutions at a time and thus requires many iterations until a final design is determined. A new approach which has repeatedly been validated in the early phase of automotive development (Fender et al., 2013; Eichstetter et al., 2014; Zimmermann and Wahle, 2015) is the identification of so called Solution Spaces (Lehar and Zimmermann, 2012; Zimmermann and Edler von Hoessle, 2013; Königs and Zimmermann, 2016; Zimmermann et al., 2017). Based on the description of functional interdependencies multiple requirements to individual design variables are considered simultaneously in a multidimensional design space (Figure 1 (b)). 


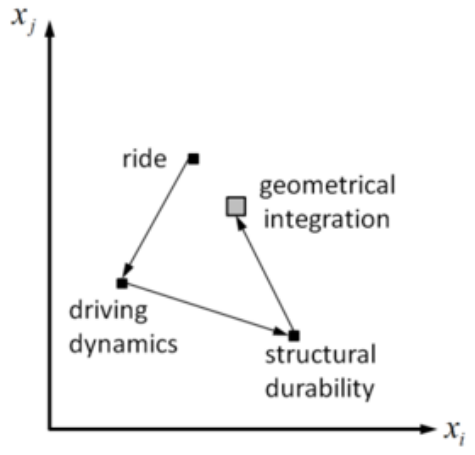

(a) Point based design

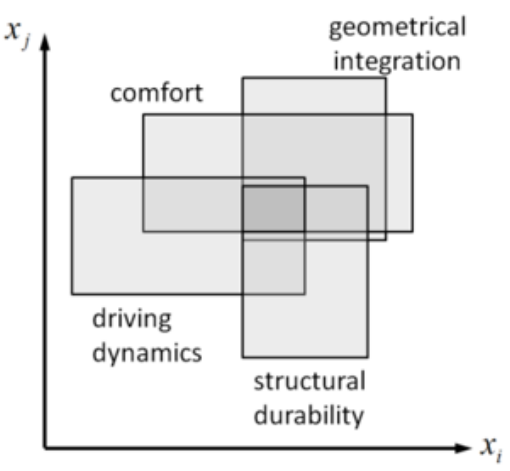

(b) Solution Space design

Figure 1. Reducing iteration in the functional layout through the identification of Solution Spaces, (from Eichstetter et al., 2014)

Whereas point based design is characterized by a discipline specific optimization of design variables which have to be adjusted subsequently due to contradictory requirements in other disciplines, the method of Solution Space design aims at an evaluation of multiple functional requirements and the identification of a Solution Space where all different requirements are fulfilled. Unlike classical optimization methods aiming for the identification of one best solution, Solution Space design aims at finding a maximum range for design variables within each of which will eventually satisfy all requirements. Taking into consideration that the layout of vehicles is subject to requirements such as the customization of the product (e.g. different types of engines or transmissions) or the standardization of interfaces to allow for a modular use of components, it becomes clear that seeking only one optimal solution is less expedient than considering a robust design for a wide range of variants.

\subsection{Resolving of goal conflicts based on the identification of Solution Spaces}

The evaluation of functional system behaviour requires models which depict the functional relations between input variables and the resulting output. Such models can include both variables which are seen as invariable and design variables which are subject to the functional layout. Figure 2 depicts a simplified (qualitative) model about the functional relations of Design Variables (Positions of Engine Mounts) and Constant variables (Mass and Center of Gravity of the Engine-Transmission Unit). In the presented example the positions of the Engine Mounts have to be chosen in a way that the load constraints of the mounts are not violated and that - based on the individual loads - the requirement of the load ratio is satisfied.

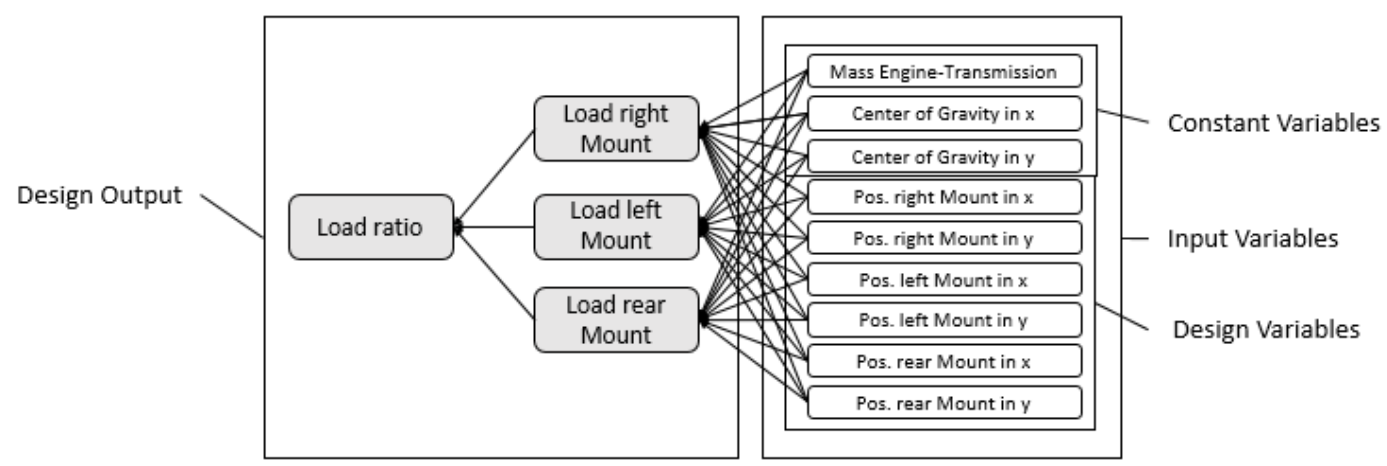

Figure 2. Functional interdependencies between Input Variables and Design Output

In order to evaluate the design output based on the design variables it requires the description of the functional interdependencies in mathematical-physical surrogate models (Poulain et al., 2018). Upon the sampling of Design Variables (indicated by the abscissa and ordinate) a design space is generated comprising a set of design points (Figure 3). The range within which design Variables can vary depends on the requirements of each design variable. Based upon the considered functional requirements the 
design points are evaluated and either categorized as good designs (green points) fulfilling the requirements or bad designs (red points) at least not fulfilling one requirement. The design space will thus contain areas of feasible designs - the Solution Space - and regions of non-feasible designs. The aim of the approach is to find the largest possible Solution Box within the Solution Space. The rectangular shape of the box allows for a decoupling of design variables in a way that whenever design variables are chosen within the Solution Box Range (LI) it will return a feasible design output.

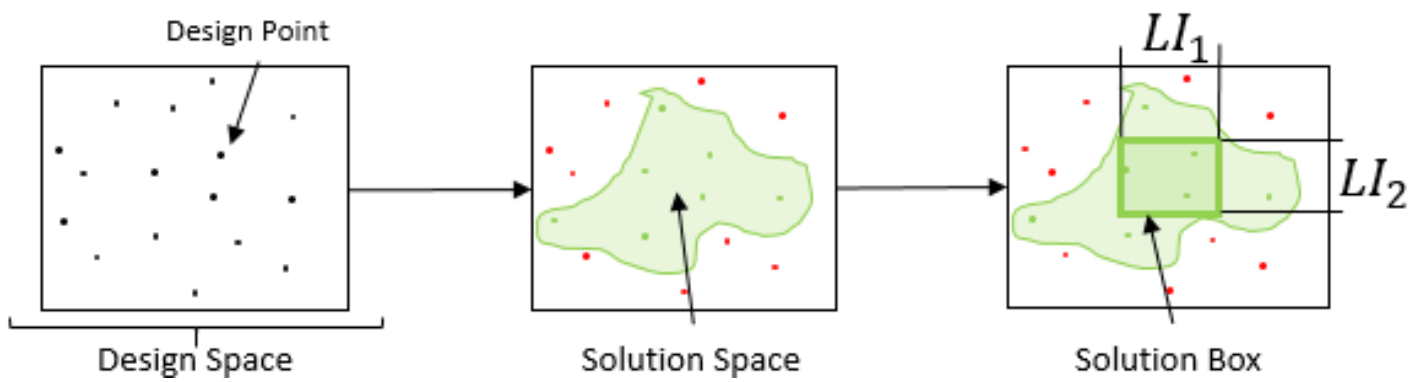

Figure 3. Exploration of Design Space to identify Solution Space and Solution Box

In this simplified example only two design variables and one functional requirement are considered. Figure 4 shows the Solution Space analysis to the problem which was schematically described in Figure 2. In this scenario four functional requirements and six design variables were considered. Green Design points indicate feasible solutions whereas differently coloured points violate the corresponding requirements.
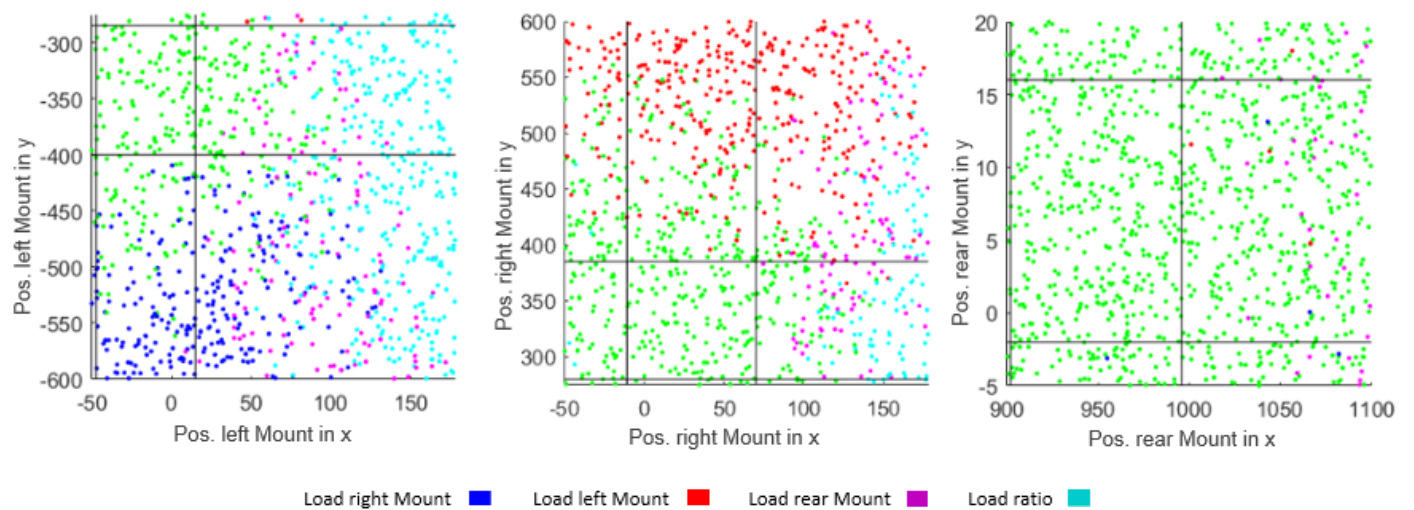

Load right Mount

Load left Mount

Load rear Mount

Figure 4. Prototypical implementation of Solution Space Approach

Also it is possible to evaluate multiple configurations for the same scenario. Figure 5 shows the consideration of different Engine-Transmission Units. Each of the configurations has a different weight and centre of gravity. Hence each configuration will have their own Solution Box for the same layout scenario.
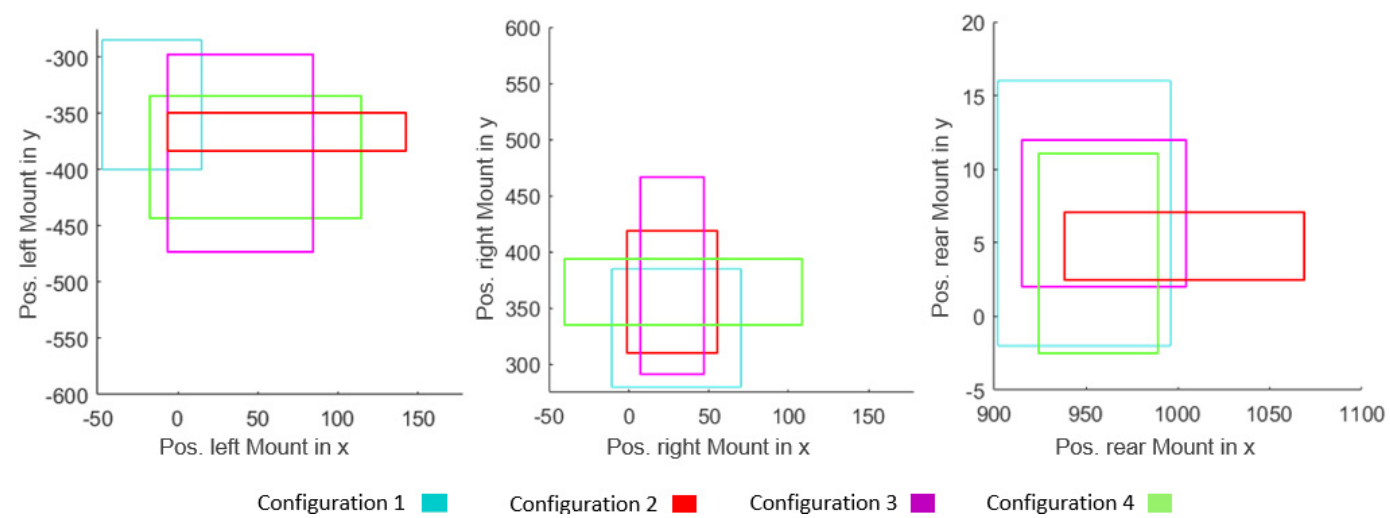

Figure 5. Solution Boxes for different Configurations 
For design problems which have to consider different configurations a Solution Box resulting from the overlapping of the configuration specific Solution Boxes will return design variable ranges within which feasible solution can be generated.

\section{Fostering transparency in engineering processes with a Parameter Management Approach}

\subsection{Challenges in the management of Engineering Information}

In order to be able to synthesize a decomposed system represented by various models to one feasible fully integrated product model it is necessary that models are co-ordinated to allow for a consistent integration. This means that information about the models / respectively model information has to be exchanged continuously throughout the development process. Due to strongly heterogeneous formats of models/Engineering Objects the automation of such tasks is very cumbersome and requires the use of tools able to manage formalized model-interdependencies (Königs et al., 2012). Hereby reported challenges in tools managing interdependency-models are especially

- High modelling effort to formalize interdependencies

- High effort to keep interdependencies up-dated and consistent

- Instantiation and adaption of the models

- Integration of such tools into existing processes and an existing IT-environment

- Instruction and support of users

- Discrepancy between effort and benefit

Ostermayer (2001) and Karniel and Reich (2011) further address a lack of mechanisms and services in PDM systems to support the exchange of updated product information. From an industrial perspective in the automotive development this deficiency in PDM systems is further intensified through

- Limited access to Engineering Objects for Individuals due to an elaborated user and rights management which is bound to organizational structures

- Lack of a compulsory and coordinated procedures in the management of Engineering Objects in the early phases which complicates the retrieval and distribution of model information

- Required knowledge about the context of engineering data in order to accurately interpret it.

The mentioned challenges in the handling of tools capable of managing model information and interdependencies as well as current practices in industry complicate their implementation and application. In consequence such tasks are mostly executed by individuals often leading to incompatible stand-alone solutions with very restricted scope or manual management and documentation in Excel sheets and PowerPoint presentation.

\subsection{Key Functionalities of Parameter Management}

The approach of Parameter Management as described by Toepfer and Naumann (2017a, b) which was implemented as a productive application at Daimler AG, Germany focuses on the management of engineering parameter as quantifiable and model-specific characteristics. Based on models as omnipresent concepts of pragmatism and reduction which represent certain system aspects (Stachoviak, 1973 ) it is the aim of the approach to manage model information as well as information about models and to make this information retrievable and exchangeable.

As quantifiable characteristics, Engineering Parameters can describe all entities of a system and are integral parts of Engineering Objects (Toepfer and Naumann, 2017b). Based on the retrieval of parameter information in models and the models' meta-information, contextualized parameter information can be retrieved from the PDM System and instantiated in a parameter management application (Toepfer and Naumann, 2016). A parameter is thus always instantiated through the assignment to a product-related context. Based on the context-specific instantiation of parameters it is also possible to assign target values or respectively target-value ranges to parameters (Figure 6). 


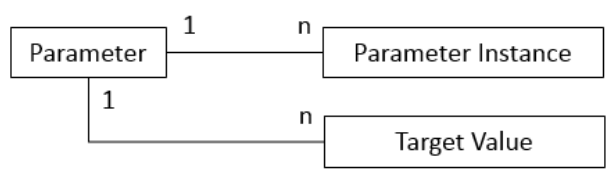

Figure 6. Instantiation of Parameters and Target Values

Actual parameter values can thus be continuously checked for compliance of defined target values throughout the development process and violations easily be identified.

\subsection{Fostering the Information Flow based on Active Chains}

With the concept of so called "Active Chains" (Vester, 2002) Toepfer and Naumann (2017b) suggested an approach to foster transparency of changes and the communication of changes in Engineering Objects based on Engineering Parameters. Active Chains therefore describe a self-customizable interdependency model which unlike many approaches do not require the semantic description of interdependencies. An Active chain thus simply comprises an aggregation of parameters relevant for the user. When one of the parameters bound to an active chain encounters a change or violates its target value or range, all other parameters in that active chain can be informed about the change or the violation respectively.

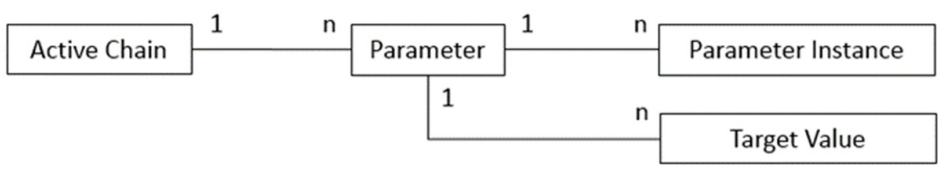

Figure 7. Aggregation of Parameters in an Active Chain

Although active chains can depict functional dependencies in a qualitative manner it is not possible to draw conclusions about how parameters affect one another. Also it is not possible to draw a conclusion how parameters should be chosen in order to fulfil various - often conflicting - functional requirements. The active chain depicted in Figure 8 shows the functional dependencies between the engineering parameters from the example used in Figure 2.

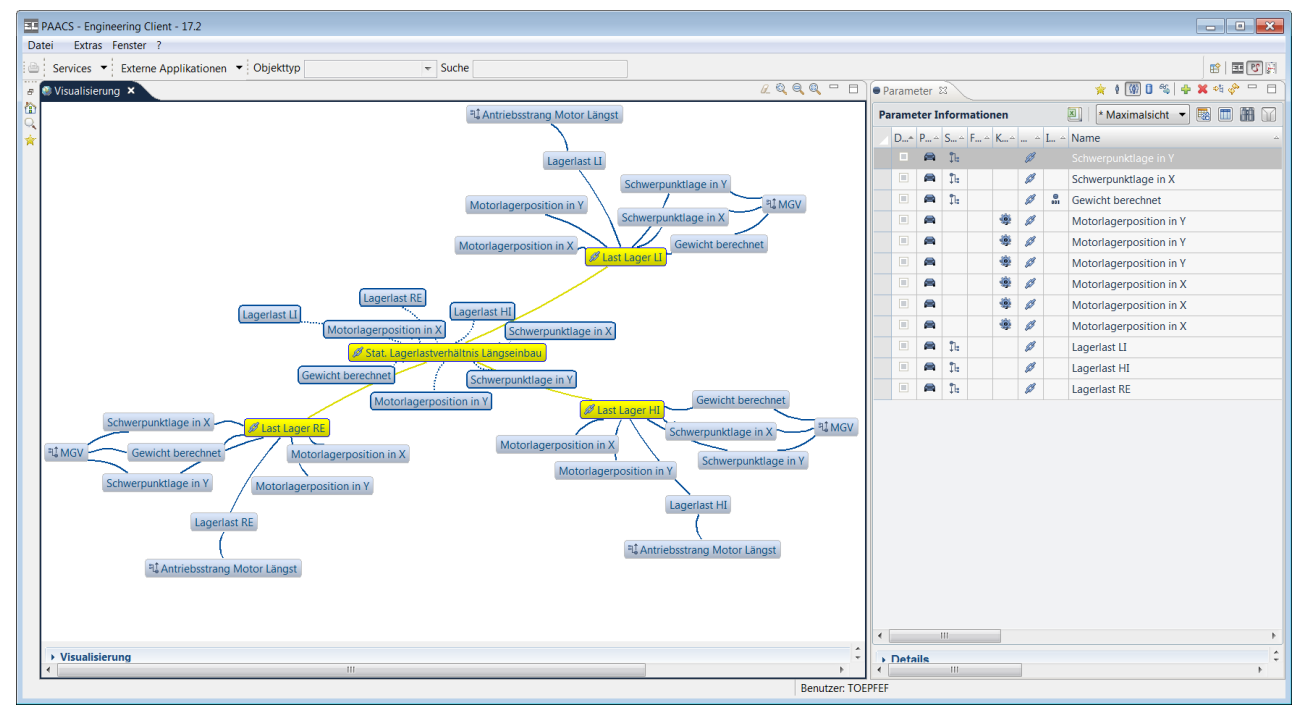

Figure 8. Active Chains with functional and structural dependencies in the Parameter Management Application

Both, the knowledge about how parameters influence one another and the knowledge about within which range parameters should be chosen to generate a feasible design output are results of the Solution Space approach. The concept described in Section 4 therefore aims at integrating this knowledge about functional interdependencies and functional feasibility into the parameter management approach in order to make it retrievable for a broad range of engineers. 


\section{Integrating the formalized knowledge about functional interdependencies into a Parameter Management Approach}

The concept that is proposed in this section is currently implemented as a prototype based on the previously described approach of Parameter Management and on the Solution Space approach described in Section 2. This section comprises both previously introduced terms of Parameters, Parameter Instances, Target Values and Target Ranges for the approach of Parameter Management (Section 3) and the terms Design Input/Output, Design Variables and Constant Variables for the Solution Space approach (Section 2). For reasons of clarity and their overlapping nature only the notation of Parameter Management will be used in this section. Input Variables and resulting Design Output are thus both referred to as Parameters. As Parameters are generic objects which are instantiated with a temporary constant value, Constant Variables are considered as Parameter Instances. Design Variables contain a permissible range within which they can be varied. This range can thus be defined through a Parameter Target-Value Range (Figure 9).

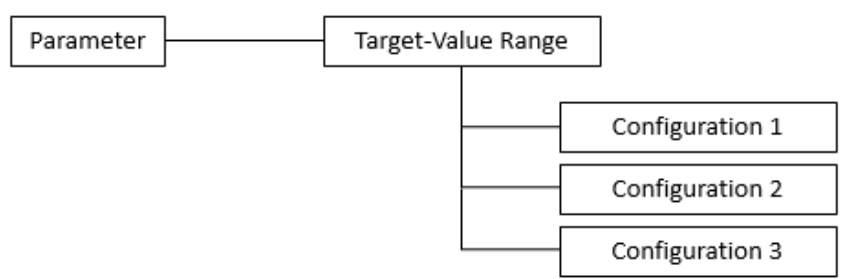

Figure 9. Configuration specific Target-Value Ranges as input for the Design Space Exploration

As outlined before Target-Value Ranges can be individually defined for different configurations of a product. However, this configuration-specific target value does not include considerations about possible functional requirements to the particular parameter. It is thus the aim to integrate this knowledge into the target-value range to allow the user to draw conclusions about how the parameter should be chosen to fulfil particular functional requirements. The formal prerequisite for combining the approach of Solution Space analyzation and parameter management is that the handled objects and the mechanisms to formalize interdependencies are compatible among each other to ensure consistency. Therefore the concept of active chains has to be able to depict the functional relations within and between mathematical models by means of aggregation and poly-hierarchy.

Through the consideration of configuration specific target values and Parameter Instances the mathematical model used in the Solution Space approach can be instantiated for configuration specific evaluations (compare Figure 5). Based upon an instantiated mathematical model additional requirements can be added to the Solution Space exploration (compare Figure 1(b)). For this, multiple mathematical models undergo a model combination defining a layout scenario for which the Solution Space is explored (Figure 10).

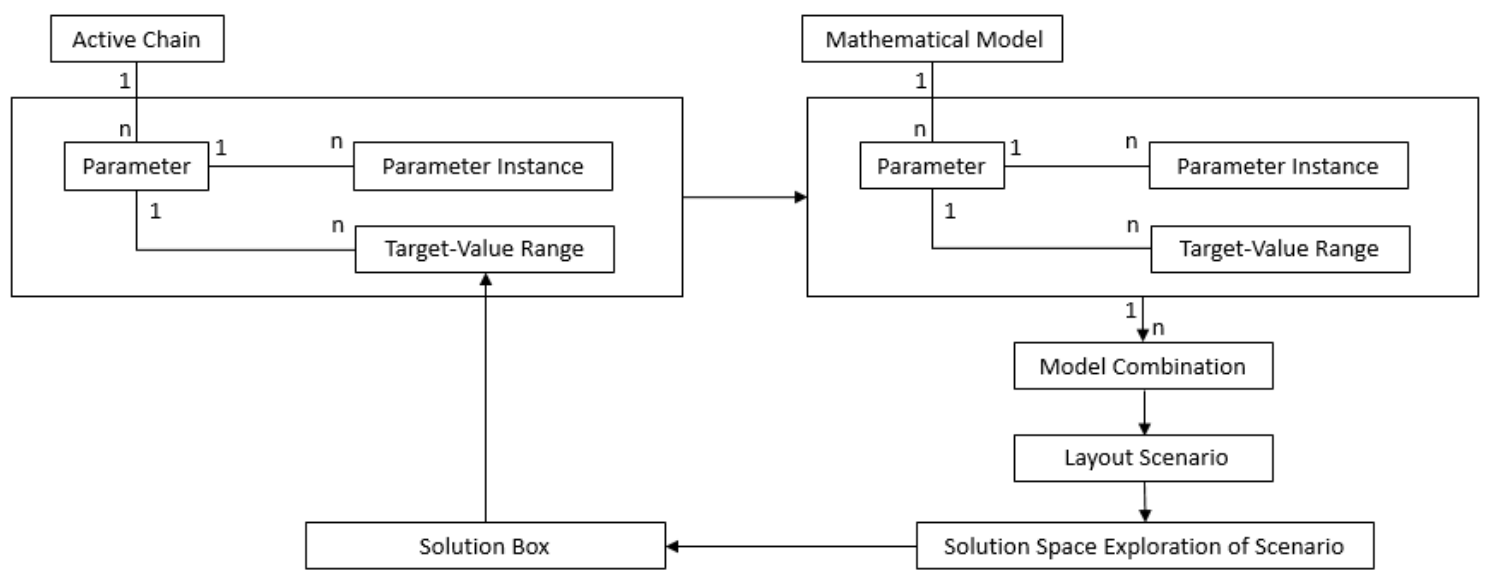

Figure 10. Interaction between the Interdependency Models of Active Chains and Surrogate Models of the Solution Space Approach 
The Solution Box which is retrieved as a result of the Solution Space exploration thus contains information about the configurations and the model combination which have been considered and allow a backward mapping to the layout problem and the underlying mathematical models. As the Solution Box returns a lower and upper bound it can be integrated into the Target-Value Management of the Parameter Management Approach. Figure 11 shows the resulting integration of Solution Space TargetValue Ranges into the configuration specific Target Value Management.

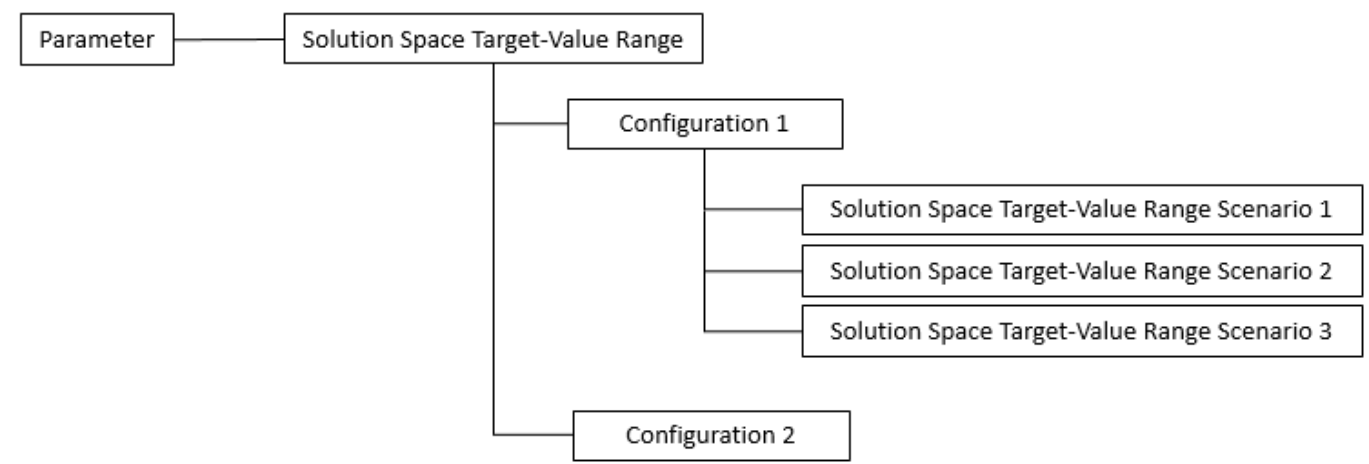

Figure 11. Integration of Solution Space Target-Value Ranges into the Target-Value Management of Parameters

Solution Space Target-Value Ranges describe the range in which - according to the Solution Space exploration - a parameter should be chosen in order to retrieve a feasible design for the layout scenario which has been considered. Based on model combinations and resulting layout scenarios multiple Solutions Space Target-Value Ranges are available for one configuration specific target value.

\section{Outline}

Communication and coordination are essential activities in engineering to allow for a consistent development of complex products. These activities require the management of interdependencies between Engineering Objects by means of appropriate tool support for once the complexity is not manually manageable anymore. Formalizing the knowledge of such dependencies however usually requires expert tools and expert knowledge and is thus not retrievable for a broad range of engineers involved in the development process.

\subsection{Summary}

The concept that has been discussed in this paper aimed at answering the research question of how the knowledge of functional interdependencies and the knowledge about feasible solutions can be formalized and made retrievable in complex engineering processes. This paper therefore presented two different approaches of managing interdependencies differing in scope, methodology and their informational content.

The first approach which was described is based on the quantitative description of functional interdependencies and allows for a solving of multidimensional goal conflicts in the functional layout. Upon the evaluation of the design space which describes all possible solutions of the interrelated functions, areas of feasible solutions, so called Solution Spaces are determined. Accordingly it is possible to draw conclusions about permissible ranges of input parameters in order to ensure functional feasibility in multidimensional layout problems. As the approach requires the formalization of functional interdependencies in mathematical models the exact functional behaviour is described.

The second approach allows for a management of qualitative interdependencies based on Engineering Parameters as integral parts of EOs, particularly addressing the challenge of transparency about the occurrence of changes in Engineering Objects and the transparency about the propagation of changes. The approach especially addresses the questions of how engineering information can be effectively monitored throughout the development process and how information about changes can be purposefully 
distributed among engineers. With the modelling of active chains users can build their own interdependency networks based on parameters which are important for their processes and work.

With the integration of Solution Space Target-Value Ranges into the Parameter Management Approach every parameter that is subject to a multidisciplinary layout problem and potential goal conflicts can be evaluated regarding the underlying layout scenarios. For such parameters it is thus possible to draw a conclusion about within which range the parameter should be chosen in order to fulfil the functional requirements which have been considered in the Solution Space exploration. Combined with the concept of active chains and the build upon information flow such evaluations can be distributed effectively within the engineering organization.

\subsection{Discussion}

The concept marks a promising approach into the integration of knowledge about functional interdependencies as well as the knowledge about feasible solutions into a tool that does not require discipline specific expert knowledge. This knowledge previously only accessible for experts can thus be distributed among non-experts and be used for the decision making in product development to obtain validated robust solutions. However, as with any new approach it needs a critical reflection in terms of risks and opportunities as well as a thoughtful integration into current processes. User acceptance therefore is a main aspect for the successful rollout of new tools. For users, already struggling with complex decision-making situations and a lack of transparency about changes in a very time-critical environment, it is clear that a new approach should be easily adaptable and easy to integrate into present processes. Despite the common awareness about challenges and the necessity for new approaches and tools, their implementation is still seen critically by most users. It is thus essential to design tools as intuitive and flexible as possible. Benefits of new tools and their functionalities have to significantly outrun the effort of using and adapting them. It is therefore essential to offer support, training on the tool and training material. Further requirements and factors for the acceptance of a broadly accessible and server-based tool are general performance, stability, susceptibility to errors, data-consistency, bugfixing and maintenance. The benefits of the approaches and the discussed integration of the SolutionSpace Approach into Parameter Management are higher transparency and communication about changes, the function-driven coordination of multidisciplinary layout problems which can accelerate the process of finding feasible solutions and the consideration of discipline specific expertise based upon a common understanding of engineering parameters which are subject to multidisciplinary goal conflicts.

\section{References}

Bitzer, M., Eigner, M. and Langlotz, M. (2007), “Management Decision Support by PLM Solutions”, Proceedings of the 16th International Conference on Engineering Design, Paris.

Browning, T.R. (2001), "Applying the Design Structure Matrix to System Decomposition and Integration Problems: A Review and New Directions", IEEE Transactions on Engineering Management, Vol. 48 No. 3 , pp. 292-306. https://doi.org/10.1109/17.946528

Dickerson, E.C. and Mavris, D. (2013), "A brief history of Models and Model Based Systems Engineering”, IEEE Systems Journal, Vol. 7 No. 4, pp. 581-592.

DIN 2330 (1996), Begriffe und Benennungen, Urs Engeler Editor, Basel, Weil am Rhein, Wien.

Dörner, D. (2003), Die Logik des Misslingens, 14th ed., Rowohlt Verlag, Reinbeck.

Ehrlenspiel, K. and Meerkamm, H. (2013), Integrierte Produktentwicklung, Carl Hanser Verlag, München, Wien.

Eichstetter, M., Redeker, C., Müller, S., Kvasnicka, P. and Zimmermann M. (2014), "Solution Spaces for damper design in vehicle dynamics", 5th International Munich Chassis Symposium, Munich, Germany. https://doi.org/10.1007/978-3-658-05978-1_10

Estefan, J.A. (2007), Survey of Model Based Systems Engineering. [online] INCOSE MBSE Focus Group. Available at: omgsysml.org

Faisst, K.G. and Dankwort, C.W. (2007), "New Extended Concept for the Usage of Engineering Objects and Product Properties in the Virtual Product Generation Process", Proceedings of the 16th International Conference on Engineering Design, Paris.

Fender, J., Duddeck, F. and Zimmermann, M. (2013), "On the Calibration of Simplified Vehicle Crash Models", Structural and Multidisciplinary Optimization, Vol. 49 No. 3, pp. 455-469. https://doi.org/10.1007/s00158013-0977-7. 
Gopsill, J.A., Snider, C., McMahon, C. and Hicks, B. (2016), “Automatic generation of design structure matrices through the evolution of product models", Artificial Intelligence for Engineering Design, Analysis and Manufacturing, Vol. 30 No. 4, pp. 424-445, https://doi.org/10.1017/S0890060416000391

Haberfellner, R., de Weck, O., Fricke, E. and Vössner, S. (2012), Systems Engineering, Orell Füssli Verlag, Zürich Hacker, W. (2002), Denken in der Produktentwicklung, Rainer Hampp Verlag.

Karniel, A. and Reich, Y. (2011), Managing the Dynamics of New Product Development Processes, Springer. https://doi.org/10.1007/978-0-85729-570-5

Königs, S. and Zimmermann, M. (2016), "Resolving conflicts of goals in complex design processes - application to the design of engine mount systems", Proceedings of 7th International Munich Chassis Symposium, https://doi.org/10.1007/978-3-658-14219-3 14

Königs, S.F., Beier, G., Figge, A. and Stark, R. (2012), "Traceability in Systems Engineering - Review of Industrial Practices, State-of-the-art Technologies and New Research Solutions", Advanced Engineering Informatics, Vol. 26 No. 4, pp. 924-940. https://doi.org/10.1016/j.aei.2012.08.002

Lehar, M. and Zimmermann, M. (2012) "An inexpensive estimate of failure probability for high-dimensional systems with uncertainty", Structural Safety, Vol. 36-37, May-July 2012, pp. 32-38. https://doi.org/10.1016/j.strusafe.2011.10.001

Naumann, T. (2005), Adaptives Systemmanagement, PhD thesis, Universität Magdeburg.

Naumann, T. (2016), "Theory of Social Systems Engineering”, Proceedings of the 14th International Design Conference, Dubrovnik, Croatia.

Ostermayer, R. (2001), Pragmatisch-Situative Wissensrepräsentation - ein Baustein für das Wissensmanagement, $\mathrm{PhD}$ thesis, Universität Karlsruhe.

Poulain, B., Naumann, T., Le Cardinal, J. and Anderer, J. (2018), “A Metaheuristic for Solution Space Modelling”, Proceedings of the 14th International Design Conference, Dubrovnik, Croatia.

Ropohl, G. (2009), Allgemeine Technologie, Universitätsverlag Karlsruhe.

Stachoviak, H. (1973), Allgemeine Modelltheorie, Springer Verlag, Wien; New York. https://doi.org/10.1007/9783-7091-8327-4

Toepfer, F. and Naumann, T. (2016), "Management of Vehicle Architecture Parameters", Proceedings of the 14th International Design Conference, Dubrovnik, Croatia.

Toepfer, F. and Naumann, T. (2017b), "Towards Cross-Linked Development of Highly Complex Products", Proceedings of the 21st International Conference on Engineering Design, ICED 2017, Vancouver, Canada, 21-25 August 2017.

Toepfer, F. and Naumann, T. (2017a), "Parameter Management, a Novel Approach in Systems Engineering", Proceedings of the 6th International Conference on Research into Design, Guwahati, India. https://doi.org/10.1007/978-981-10-3518-0_34

Vester, F. (2002), Die Kunst vernetzt zu denken, Ideen und Werkzeuge für einen neuen Umgang mit Komplexität, Dt. Taschenbuch Verlag, München.

Zimmermann, M., Königs, S., Niemeyer, C., Fender, J., Zeherbauer, C. et al. (2017), "On the design of large systems subject to uncertainty”, Journal of Engineering Design, Vol. 28 No. 4, pp. 233-254. https://doi.org/10.1080/09544828.2017.1303664

Zimmermann, M. and Wahle, M. (2015), "Solution Spaces for Vehicle Concepts and Architectures", 24th Aachen Colloquium Automobile and Engine Technology.

Zimmermann, M. and Edler von Hoessle, J. (2013), "Computing Solution Spaces for robust design", International Journal for Numerical Methods in Engineering, Vol. 94 No. 3, pp. 290-307. https://doi.org/10.1002/nme.4450

Ferdinand Toepfer, M.Sc.

Daimler AG, Engineering IT

Hanns-Klemm Str.5, 71034 Böblingen, Germany

Email: ferdinand.toepfer@daimler.com 